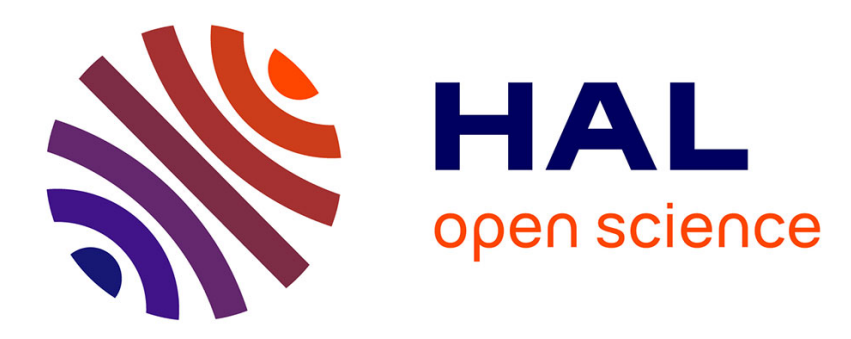

\title{
Walking gait of a planar bipedal robot with four-bar knees
}

\author{
Arnaud Hamon, Yannick Aoustin
}

\section{To cite this version:}

Arnaud Hamon, Yannick Aoustin. Walking gait of a planar bipedal robot with four-bar knees. Movement \& Sport Sciences - Science \& Motricité, 2013. hal-00760889

\section{HAL Id: hal-00760889 \\ https://hal.science/hal-00760889}

Submitted on 4 Dec 2012

HAL is a multi-disciplinary open access archive for the deposit and dissemination of scientific research documents, whether they are published or not. The documents may come from teaching and research institutions in France or abroad, or from public or private research centers.
L'archive ouverte pluridisciplinaire HAL, est destinée au dépôt et à la diffusion de documents scientifiques de niveau recherche, publiés ou non, émanant des établissements d'enseignement et de recherche français ou étrangers, des laboratoires publics ou privés. 


\title{
Walking gait of a planar bipedal robot with four-bar knees
}

\author{
Hamon Arnaud, and Aoustin Yannick (Corresponding author) \\ * L'UNAM, IRCCyN, UMR CNRS 6597, \\ CNRS, École Centrale de Nantes, Université de Nantes \\ 1, rue de la Noë, BP 92101. 44321 Nantes, France \\ [arnaud.hamon,yannick.aoustin]@irccyn.ec-nantes.fr
}

\begin{abstract}
The design of a knee joint is a key issue in robotics and biomechanics to increase the compatibility between prostheses and human movements and to improve the performances of the bipedal robot. We propose a novel design for the knee joint of a planar bipedal robot, based on a four-bar linkage. The advantage of this structure is to produce a translation of the Instantaneous Center of Rotation (ICR) of the knee joint like in the human case. We propose to study the sthenic criterion of a bipedal robot using four-bar knee joint during a walking gait. This walking gait is a succession of finite time double support phases, single support phases and impacts. During the double support phase, both feet rotate. This phase is ended by an impact on the ground of the toe of one foot, the other foot taking off. The single support phase is ended by an impact of the swing foot heel, the other foot keeping contact with the ground through its toe. A parametric optimization problem is presented for the determination of the parameters corresponding to the optimal cyclic walking gait. The main contribution of this paper is to show the influence of double support phases for cyclic stable walking gaits with this novel bipedal robot.
\end{abstract}

\section{Introduction}

The researchers in biomechanics have improved a lot the understanding of the human lower limb and especially the knee joint [35] and the ankle joint [27]. Indeed, these two joints have a complex architecture formed by non symmetric surfaces. This common architecture of the knee has not really changed the last 300 million years despite an important diversity of the functional need [9]. The motions of the femur with respect to the tibia are limited due to many ligaments and the patella. In addition to the flexion in the sagittal plane, there is an internal rotation with a displacement of the Instantaneous Center of Rotation $(I C R)$ of the knee joint and a posterior translation of the femur on the tibia. The mobility of the knee joint consists of three important rotations and two translations, which are the flexion-extension, adduction-abduction, and external-internal rotations, and translation in the sagittal plane, see [10]. These motions are guided by the cruciate ligaments and the articular contacts, see [15] and [35]. Then they cannot be only represented by one or two revolute joints. Different studies have confirmed these results by an observation of the motions of the human knee in the 3D space [26].

To reproduce the movement of the knee several models exist. Freudenstein et al [14] proposed a mathematical description, based on the Euler-Savary equation and relaters concepts, of the tibiofemoral relative planar motion. The femoral condyle was approximated in [25] by a spherical joint, from measurement carried out on the posterior portion. Delp et al [8] chose an ellipse in the sagittal plane to approximate the femoral condyle. In 1974, A. Menschik [28] proposed to represent the knee joint by a crossed four-bar linkage. A classical polycentric knee is the crossed four-bar linkage [16], which is used in most prosthetic knees. This mechanism is the dual solution of the classical four-bar knee but does not have any singularity in the range of motion typically used. As a matter of fact, the kinematic singularities of the mechanism may limit its range of motion, mainly when an important knee flexion is required. The dimensions of the crossed four-bar linkage can be chosen by measuring, on a real subject: (i) the length of the anterior and posterior ligaments; (ii) the position of the crossed ligament attachments on the tibia and the femur, projected on the sagittal plane in the maximum extension position [12]. Authors in [11] developed a dynamic model for the lower extremity capable of estimating forces generated in the knee. The proposed model considers on a four-bar mechanism in the knee, a spherical joint in pelvis and a revolute one in the ankle. Authors proved experimentally through a force platform and high-speed cameras that the crossed four-bar mechanism could reproduce the knee flexion-extension and the two main translations. As a consequence for the knee the adduction-abduction and external-internal rotations are not modeled. However, these angles are relatively small when compared with the flexion-extension angle.

Roboticists have come up with new and better bipedal robots recently. For instance, HRP-2LR [23] and RABBIT [5], which are able to run and and to be dynamically stable. A. Grishin et al. [19] focused 
on the design of a bipedal robot with telescopic legs. T. Yang et al. [36] used a compliant parallel knee to improve the walking motion. Some authors also dealt with the walking and running gaits using the toe rotation [22], [30], [31]. Our objective is to improve the bipedal robot performance thanks to a new design of the knee joint. Several papers deal with the bipedal robots equipped with complex knees, like G. Gini et al. [18] which used knee joints based on the human knee surfaces. F. Wang et al. [34] developed a bipedal robot with two different joints, namely, a revolute joint and a four-bar linkage. We proved in [20] that a knee based on a four-bar linkage is better than a knee designed with a revolute joint in terms of energy consumption. With a good choice of the length of the links of the four-bar and its cross configuration the flexion of the knee joint is usually not too limited with the kinematic singularities.

This paper aims to study the performance of a planar bipedal robot, equipped with knees based on four-bar linkages, during a complex walking gait. This gait is composed of finite time double support phases, single support phases and impacts. During the double support phase, both feet rotate. This phase ends with a contact of one toe with the ground while the other foot is taking off. The single support phase ends with an impact of the swing foot heel, the toe of the other foot being in contact with the ground. We also present the dynamic model of the planar bipedal robot whose knees are composed of a four-bar linkage. We developed a parametric optimization method to define a set of optimal reference trajectories for both cyclic gaits. We use this optimization process to study the effect of the double support for cyclic walking gaits.

This paper is organized as follows. Section 2 presents the novel planar bipedal robot whose knees are based on four-bar linkages and its dynamic models. Section 3 deals with the trajectory planning. Section 4 proposes numerical results about the evaluation of the energy consumption as a function of the bipedal robot velocity. Finally, Section 5 presents some conclusions and future works.

\section{Presentation of the bipedal robot with knees composed of a four-bar linkage}

Let us introduce the bipedal robot, which is depicted in Figure 1. Table 1 gathers the physical data of the biped, which are taken from Hydroid, a humanoid bipedal robot [32].

\begin{tabular}{|c|c|c|c|c|}
\hline & Mass $(k g)$ & Length $(m)$ & Inertia $\left(\mathrm{kg} \cdot \mathrm{m}^{2}\right)$ & $\begin{array}{l}\text { Center of } \\
\text { mass }(m)\end{array}$ \\
\hline foot & $m_{f}=0.678$ & $\begin{array}{l}L_{p}=0.207 \\
l_{p}=0.072 \\
H_{p}=0.064\end{array}$ & 0.002 & $\begin{array}{l}s_{p x}=0.0135 \\
s_{p y}=0.0321\end{array}$ \\
\hline shin & 2.188 & 0.392 & 0.028 & $s_{1}=s_{4}=0.169$ \\
\hline thigh & 5.025 & 0.392 & 0.066 & $s_{2}=s_{3}=0.169$ \\
\hline trunk & 29.27 & 0.403 & 0.815 & $s_{5}=0.192$ \\
\hline $\begin{array}{l}\text { four-bar } \\
\text { knee }\end{array}$ & $m_{d}=1.2$ & \multicolumn{3}{|c|}{$\begin{array}{l}l_{a}=A B=0.029 \mathrm{~m} \\
l_{b}=B C=0.035 \mathrm{~m} \\
l_{c}=C D=0.015 \mathrm{~m} \\
l_{d}=A D=0.025 \mathrm{~m}\end{array}$} \\
\hline
\end{tabular}

Table 1: Physical parameters of the bipedal robot.

The dimensions of the four-bar linkage are chosen with respect to the human characteristics measured by J. Bradley et al. through radiography in [2] and in accordance with the hydroid geometrical parameters to avoid any singularity in the knee joints during the walking gaits.

Figures 1; 2(a) and 2(b) depict the bipedal robot under study and its four-bar knee linkage. Figure 2 (a) represents the four-bar knee linkage. The angular variable $\alpha_{1}$ is the actuated variable of the four-bar linkage.

The bipedal robot is equipped with two closed-loop knees. Let us introduce the constraint equations solving the dynamic model [24]. Equations for knee joints 1 and 2 are similar. For a sake of clarity we consider knee joint 1 only. The equations of the closed-loop geometric constraints are defined as follows:

$$
\begin{aligned}
& a \cos q_{1}-b \sin q_{g_{11}}+c \cos q_{2}+d \sin q_{g_{12}}=0 \\
& a \sin q_{1}+b \cos q_{g_{11}}-c \sin q_{2}-d \cos q_{g_{12}}=0
\end{aligned}
$$




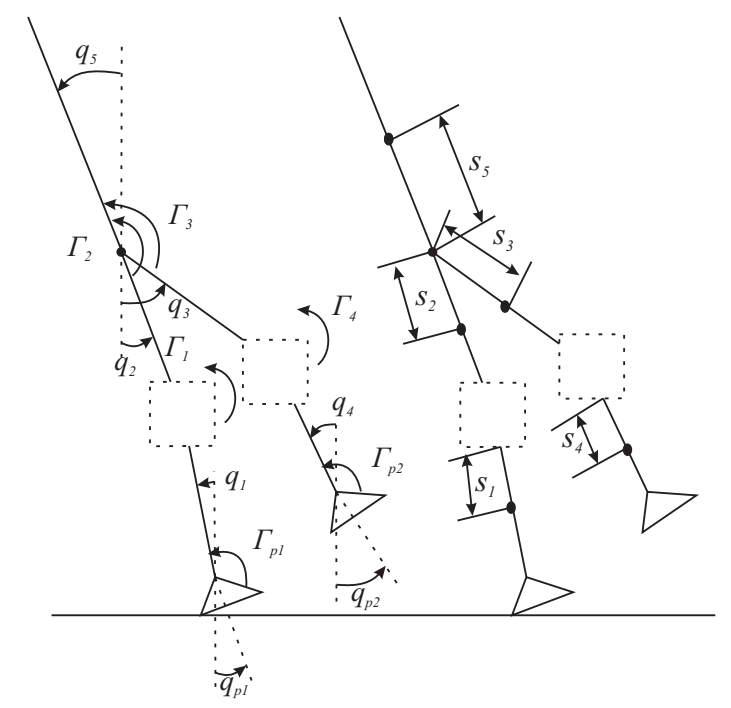

Figure 1: Schematic of a planar bipedal robot. Absolute angular variables and torques.

Their first and second time derivatives are

$$
\begin{gathered}
-a \dot{q}_{1} \sin q_{1}-b \dot{q}_{g_{11}} \cos q_{g_{11}}-c \dot{q}_{2} \sin q_{2}+d \dot{q}_{g_{12}} \cos q_{g_{12}}=0 \\
a \dot{q}_{1} \cos q_{1}-b \dot{q}_{g_{11}} \sin q_{g_{11}}-c \dot{q}_{2} \cos q_{2}+d \dot{q}_{g_{12}} \sin q_{g_{12}}=0
\end{gathered}
$$

and

$$
\begin{gathered}
-a \ddot{q}_{1} \sin q_{1}-b \ddot{q}_{g_{11}} \cos q_{g_{11}}-c \ddot{q}_{2} \sin q_{2}+d \ddot{q}_{g_{12}} \cos q_{g_{12}} \\
-a \dot{q}_{1}^{2} \cos q_{1}+b \dot{q}_{g_{11}}^{2} \sin q_{g_{11}}-c \dot{q}_{2}^{2} \cos q_{2}-d \dot{q}_{g_{12}}^{2} \sin q_{g_{12}}=0 \\
a \ddot{q}_{1} \cos q_{1}-b \ddot{q}_{g_{11}} \sin q_{g_{11}}-c \ddot{q}_{2} \cos q_{2}+d \ddot{q}_{g_{12}} \sin q_{g_{12}} \\
-a \dot{q}_{1}^{2} \sin q_{1}-b \dot{q}_{g_{11}}^{2} \cos q_{g_{11}}+c \dot{q}_{2}^{2} \sin q_{2}+d \dot{q}_{g_{12}}^{2} \cos q_{g_{12}}=0 .
\end{gathered}
$$

Through the virtual work principle, these constraint equations can be expressed in the dynamic model by adding the Lagrange multipliers $\mathbf{J}_{\mathbf{1}_{\mathbf{e}}}^{\mathbf{t}} \lambda$. Here $\mathbf{J}_{\mathbf{1}_{\mathbf{e}}}$ is the $2 \times 13$ Jacobian matrix such as equations (2) and (3) can be rewritten under the compact forms

$$
\mathbf{J}_{1_{e}} \dot{\mathbf{x}}=0
$$

and

$$
\mathbf{J}_{1_{e}} \ddot{\mathbf{x}}+\dot{\mathbf{J}}_{1_{e}} \dot{\mathbf{x}}=0 .
$$

and vector $\lambda=\mathbf{f}_{\mathbf{c}_{1}}=\left[f_{x_{1}}, f_{y_{1}}\right]^{\mathrm{t}}$ defines the two Lagrange multipliers vector associated to the constraint of the closed-loop linkage. These multipliers represent the exerted forces on point $A$ of the knee joint 1 (Fig. 2(a)). The generalized vector $\mathbf{x}$ is such as

$$
\mathbf{x}=\left[q_{p_{1}}, q_{p_{2}}, q_{1}, q_{2}, q_{3}, q_{4}, q_{5}, q_{g_{11}}, q_{g_{12}}, q_{g_{21}}, q_{g_{22}}, x_{h}, y_{h}\right]^{\mathrm{t}} .
$$

We apply the same principle for the knee joint 2 to obtain the dynamic model of the bipedal robot with the four-bar knees:

$$
\mathbf{A}_{e}(\mathbf{x}) \ddot{\mathbf{x}}+\mathbf{h}_{e}(\mathbf{x}, \dot{\mathbf{x}})=\left[\begin{array}{lll}
\mathbf{D}_{\Gamma_{e}} & \mathbf{J}_{1_{e}}^{\mathrm{t}} & \mathbf{J}_{2_{e}}^{\mathrm{t}}
\end{array}\right]\left[\begin{array}{c}
\boldsymbol{\Gamma}_{\mathbf{m}} \\
\mathbf{f}_{\mathbf{c}}
\end{array}\right]+\mathbf{J}_{r_{1}}^{\mathrm{t}}\left[\begin{array}{c}
\mathbf{r}_{1} \\
\mathbf{m}_{1_{z}}
\end{array}\right]+\mathbf{J}_{r_{2}}^{\mathrm{t}}\left[\begin{array}{c}
\mathbf{r}_{2} \\
\mathbf{m}_{2_{z}}
\end{array}\right]
$$

with the constraint equations:

$$
\begin{gathered}
\mathbf{J}_{r_{i}} \ddot{\mathbf{x}}+\dot{\mathbf{J}}_{r_{i}} \dot{\mathbf{x}}=\mathbf{0} \text { for } i=1 \text { to } 2 \\
{\left[\begin{array}{l}
\mathbf{J}_{1_{e}} \\
\mathbf{J}_{2_{e}}
\end{array}\right] \ddot{\mathbf{x}}+\left[\begin{array}{l}
\dot{\mathbf{J}}_{1_{e}} \\
\dot{\mathbf{J}}_{2_{e}}
\end{array}\right] \dot{\mathbf{x}}=0}
\end{gathered}
$$

$\boldsymbol{\Gamma}_{\mathbf{m}}=\left[\Gamma_{p_{1}}, \Gamma_{p_{2}}, \Gamma_{1}, \Gamma_{2}, \Gamma_{3}, \Gamma_{4}\right]^{\mathrm{t}}$ is the vector of the applied joint torques, $\left[\mathbf{r}_{i} \mathbf{m}_{i_{z}}\right]^{\mathrm{t}}$, with $i=1$ to 2 , are the resultant wrenchs of the contact efforts with the ground reaction in both feet, and $\mathbf{f}_{\mathbf{c}}=\left[\mathbf{f}_{\mathbf{c}_{1}}^{\mathrm{t}}, \mathbf{f}_{\mathbf{c}_{2}}^{\mathrm{t}}\right]^{\mathrm{t}}$. Torques $\Gamma_{1}$ and $\Gamma_{4}$ respectively actuate joint $D$ of knee 1, see (Fig. 2(a)), and the similar joint of knee 2 . where $x_{h}$ and $y_{h}$ are the hip coordinates. $\mathbf{J}_{r_{1}}$ and $\mathbf{J}_{r_{2}}$ are the $3 \times 13$ jacobian matrix of the constraint 


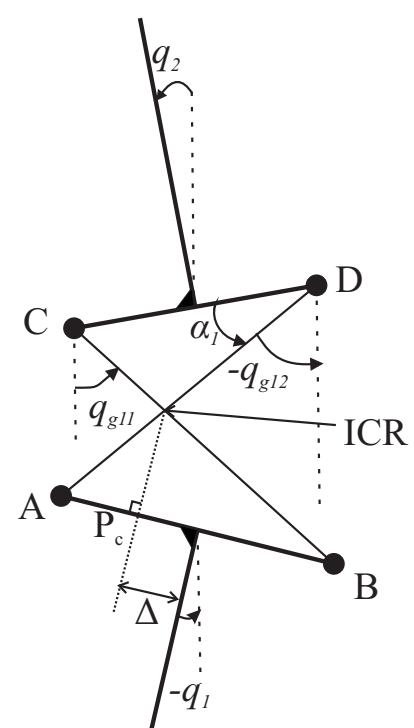

(a)

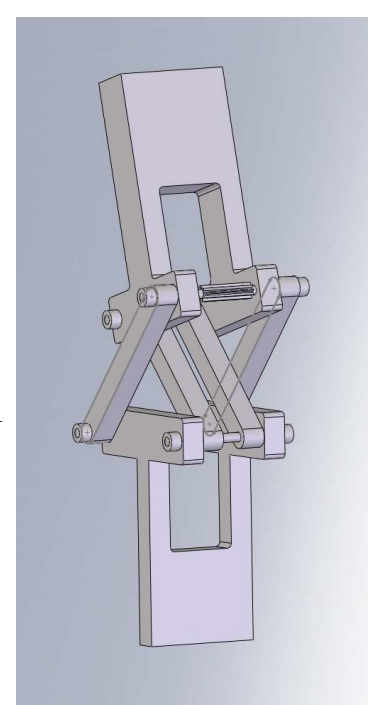

(b)

Figure 2: Details of the four-bar joint and position of the Instantaneous Center of Rotation $(I C R)$

equations in position and orientation for the two feet, respectively. $\mathbf{A}_{e}(\mathbf{x})$ is the $13 \times 13$ symmetric positive definite inertia matrix, $\mathbf{h}_{e}(\mathbf{x}, \dot{\mathbf{x}})$ is the $13 \times 1$ vector, which groups the centrifugal, Coriolis effects, and the gravity forces. $\mathbf{D}_{\boldsymbol{\Gamma}}$ is a $13 \times 6$ matrix composed of the 0 and \pm 1 given by the principle of virtual works [1].

This dynamic model (6) with constraints (7) is valid in single and double support phases. During a single support phase the ground reaction force is zero on the swing foot.

\subsection{Reduced model in single support}

The aim is to propose a dynamic model with an implicit liaison of the stance foot, here assumed to be foot 1 , with the ground to calculate the torques during the optimization process, with the knowledge of the reference trajectories for the generalized coordinates. This reduced dynamic model is only valid if the stance foot does not take off and there is no sliding during the swing phase.

Then, during the single support phase, the stance foot is assumed to remain in flat contact on the ground, i.e., there is no sliding motion, no take-off, no rotation $\left(q_{p_{1}}=0\right)$. We can use a new generalized vector $\mathbf{q}=\left[q_{p_{2}}, q_{1}, q_{2}, q_{3}, q_{4}, q_{5}, q_{g_{11}}, q_{g_{12}}, q_{g_{21}}, q_{g_{22}}\right]^{\mathrm{t}}$. The reduced dynamic model does not depend on the ground reaction force, which is applied in the stance flat-foot. The dynamic model in single support phase for the biped equipped with the four-bar knees is given by the simplification of the dynamic model (6) and (7):

$$
\mathbf{A}(\mathbf{q}) \ddot{\mathbf{q}}+\mathbf{h}(\mathbf{q}, \dot{\mathbf{q}})=\left[\begin{array}{lll}
\mathbf{D}_{\boldsymbol{\Gamma}} & \mathbf{J}_{1}^{\mathrm{t}} & \mathbf{J}_{2}^{\mathrm{t}}
\end{array}\right]\left[\begin{array}{c}
\boldsymbol{\Gamma}_{\mathbf{m}} \\
\mathbf{f}_{c}
\end{array}\right] .
$$

with the constraint matrix equation,

$$
\left[\begin{array}{l}
\mathbf{J}_{1} \\
\mathbf{J}_{2}
\end{array}\right] \ddot{\mathbf{q}}+\left[\begin{array}{l}
\dot{\mathbf{J}}_{1} \\
\dot{\mathbf{J}}_{2}
\end{array}\right] \dot{\mathbf{q}}=0
$$

Here vector $\mathbf{f}_{c}$ is such as $\mathbf{f}_{c}=\left[\mathbf{f}_{c_{1}}^{\mathrm{t}}, \mathbf{f}_{c_{2}}^{\mathrm{t}}\right]^{\mathrm{t}}$.

To define the constraints about the ground reaction, the no take-off of the stance flat-foot during the optimization process, we recall the calculation of position of the Zero Momentum Point. The resultant force $\mathbf{r}_{1}$ of the ground reaction can be calculated by applying the second Newton law at the center of mass of the biped:

$$
m \boldsymbol{\gamma}=\mathbf{r}_{1}+m \mathbf{g}
$$

where $m$ is the global mass of the biped, $\gamma=\left[\ddot{x}_{g}, \ddot{y}_{g}\right]^{\mathrm{t}}$ are the horizontal and vertical components of the acceleration for its center of mass in the world frame. $\mathbf{g}=[0,-g]^{\mathrm{t}}$ is the vector of the acceleration of the gravity. This equation allows directly to get $\mathbf{r}$ during the single support. Assuming the center of mass $G_{f}$ of the foot has for coordinates $\left(s_{p x}, s_{p y}\right)$, see Fig. 3. Let $m_{f}$ be the mass of the foot. For the planar 


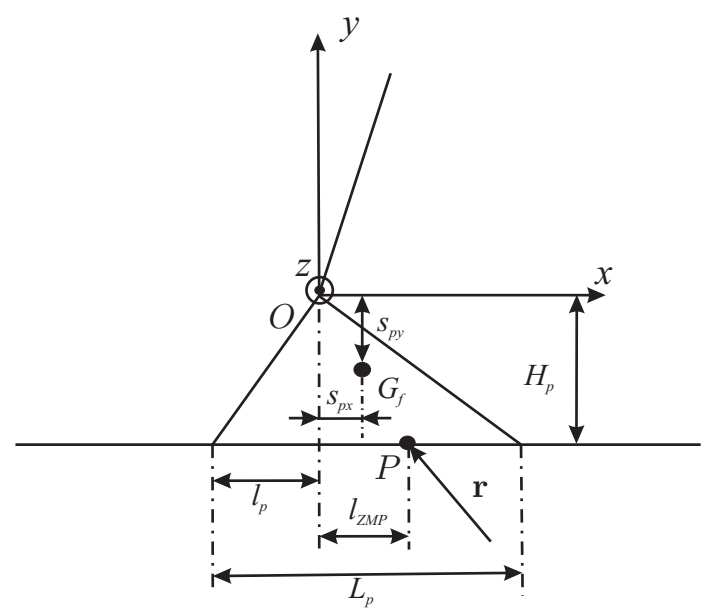

Figure 3: Details of the foot.

biped the coordinate of the Zero Moment Point $(Z M P)$, which is merged with the centre of pressure to keep the flat contact, can be obtained through the calculation of the global equilibrium of the bipedal robot around axis $z$, which gives (Vukobratovic [33]):

$$
l_{Z M P}=\frac{\Gamma_{p_{1}}+s_{p x} m_{f} g-H_{p} r_{x}}{r_{y}},
$$

To calculate the applied joint torques, which are used to obtain the sthenic criterion of the bipedal robot during the optimization process, we use the dynamic model (8).

We assume the friction effects due to the four-bar mechanism are negligible with respect to those in the gearbox of the actuators. Then only the performances of actuators are considered to study the eof volition sthenic criterion for the biped. No friction terms are included in the model.

\subsection{Impact model}

During the biped's gait, impacts occur, when the sole, the heel or the toe of the swing foot touches the ground. Let $T$ be the instant of an impact. We assume that the impact is absolutely inelastic and that the foot does not slip. Given these conditions, the ground reactions at the instant of an impact can be considered as impulsive forces and defined by Dirac delta-functions $\mathbf{r}_{j}=\mathbf{i}_{j} \delta(t-T)(j=1,2)$. Here $\mathbf{i}_{j}=$ $\left[i_{j x}, i_{j y}\right]^{\mathrm{t}}$ is the magnitudes vector of the impulsive reaction in foot $j$ (see [13]). Impact equations can be obtained through integration of the matrix motion equation (6) for the infinitesimal time from $T^{-}$to $T^{+}$. The torques provided by the actuators in the joints, Coriolis and gravity forces have finite values. Thus they do not influence the impact. Consequently the impact equations can be written in the following matrix form:

$$
\mathbf{A}_{e}(\mathbf{x}(T))\left(\dot{\mathbf{x}}^{+}-\dot{\mathbf{x}}^{-}\right)=\left[\begin{array}{ll}
\mathbf{J}_{1_{e}}^{\mathrm{t}} & \mathbf{J}_{2_{e}}^{\mathrm{t}}
\end{array}\right] \mathbf{i}_{f_{c}}+\mathbf{J}_{r_{1}}^{\mathrm{t}} \mathbf{i}_{1}+\mathbf{J}_{r_{2}}^{\mathrm{t}} \mathbf{i}_{2}
$$

Here $\mathbf{x}(T)$ denotes the configuration of the biped at instant $t=T$, (this configuration does not change at the instant of the impact), $\dot{\mathbf{x}}^{-}$and $\dot{\mathbf{x}}^{+}$are respectively the velocity vectors just before and just after an inelastic impact. Vectors $\mathbf{i}_{1}, \mathbf{i}_{2}$ and $\mathbf{i}_{f_{c}}=\left[\mathbf{i}_{f_{c 1}}^{\mathrm{t}}, \mathbf{i}_{f_{c 2}}^{\mathrm{t}}\right]^{\mathrm{t}}$ are respectively the impulsive wrenchs in both feet and the impulsive forces in both knees. To take into account of the closed-loop of the four-bar knee linkage we have to complete (12) with:

$$
\left[\begin{array}{l}
\mathbf{J}_{1_{e}} \\
\mathbf{J}_{2_{e}}
\end{array}\right] \dot{\mathbf{x}}^{+}=\mathbf{0}
$$

The velocity of the contact part of the stance foot $(j=1)$ before an impact is null.

$$
\mathbf{J}_{r_{1}} \dot{\mathbf{x}}^{-}=\mathbf{0}
$$

The swing foot $(j=2)$ after the impact becomes a stance foot. Therefore, the velocity of its contact part with the ground becomes zero after the impact,

$$
\mathbf{J}_{r_{2}} \dot{\mathbf{x}}^{+}=\mathbf{0}
$$


Generally speaking, two results are possible after the impact, if we assume that there is no slipping of the stance feet. The stance foot lifts off the ground or both feet remain on the ground. In the first case, the vertical component of the velocity of the taking-off foot just after the impact must be directed upwards. Also there is no interaction (no friction, no sticking) between the taking-off foot and the ground. The ground reaction in this taking-off leg tip must be null. In the second case, the stance foot velocity has to be zero just after the impact. The ground produces impulsive reactions (generally, $\mathbf{i}_{j} \neq 0, j=1,2$ ) and the vertical components of the impulsive ground reactions in both feet are directed upwards. For the second case, the passive impact equation (12) must be completed by one matrix equations.

$$
\mathbf{J}_{r_{1}} \dot{\mathbf{x}}^{+}=\mathbf{0}
$$

In general, the result of an impact depends on two factors: the biped's configuration at the instant of an impact and the direction of the swing foot velocity just before impact [13]. After an impact for a biped, there are two possible phases: a single support or a finite time double support. The resolution of the system composed (12), (13), (15) and eventually (16) gives the velocity vector $\dot{\mathbf{x}}^{+}$just after the impact, the impulsive reaction wrenchs $\mathbf{i}_{1}, \mathbf{i}_{2}$ and the impulsive forces $\mathbf{i}_{f_{c}}=\left[\mathbf{i}_{f_{c 1}}^{\mathrm{t}}, \mathbf{i}_{f_{c 2}}^{\mathrm{t}}\right]^{\mathrm{t}}$ relatively to the velocity vector $\dot{\mathbf{x}}^{-}$just before the impact.

To calculate the position of the $Z M P$ at the impact with flat-foot, we have to take into account of the impulsive ground reaction in the global equilibrium of the stance foot and the result is:

$$
l_{Z M P}=-\frac{H_{p} i_{j x}}{i_{j y}} .
$$

\section{Gait optimization for the cyclic walking}

\subsection{Principle}

The studied trajectories are composed of double support phases and single support phases. During the double support phase, we denote a rotation of the forward foot around the heel and a rotation of the backward foot around the toe. The double support phase is ended by the flat contact of the forward foot on the ground. The single support phase is ended by the contact of the swing foot on the toe.

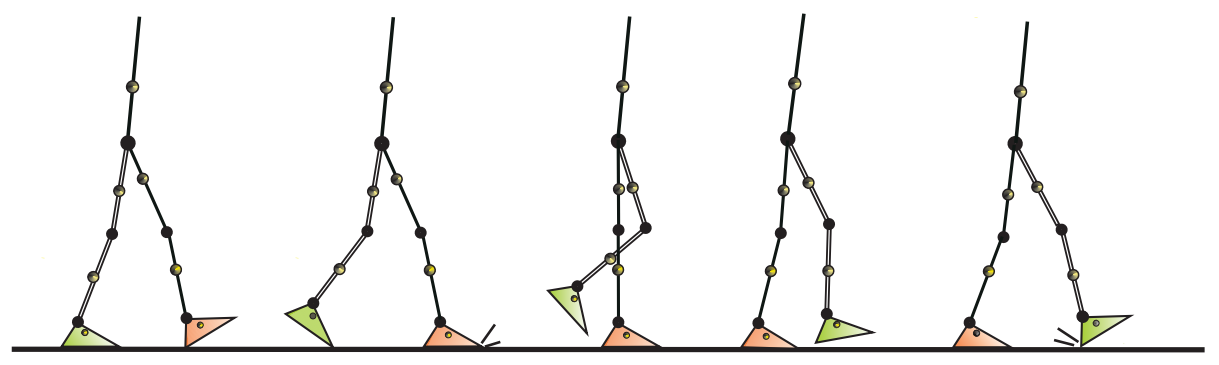

Figure 4: Presentation of the studied walking gait.

The biped with a stance flat-foot is driven by six torques. Its configuration is given in double support phase with five generalized coordinates only because the positions of the front heel and the rear toe are constant. These five independent coordinates are the orientation of feet $q_{p_{1}}$ and $q_{p_{2}}$, the orientation of one of the leg $1 q_{1}, q_{2}$ and the inclination of the torso $q_{5}$. For these coordinates, cubic spline functions $\theta_{i}(t)$, $i=1, \ldots, n_{j}=5$ are defined with an initial value in position and velocity and a final value in position and velocity, with two selected knots, $n=2$. Thus, for each joint we need to define four parameters of position and velocity to design the trajectories. The final configuration of the biped is with one flat-foot on the ground. Thus only four independent variables are necessary to define it. We use $q_{p_{2}}, q_{1}, q_{2}$ and the inclination of the torso $q_{5}$. Only five components of the joint velocities are independent during this phase, thus the final velocity can be defined with five variables at maximum. At the end of this phase from the impact, which occurs on the front toe, six initial angular velocities can be determined for the next single phase. When functions $\theta_{i}(t), i=1, \ldots, 5$ are chosen, the joint velocity and the joint acceleration can be deduced by the derivation of the polynomial function. For a given motion, the torques required could be calculated solving the inverse dynamic model (6). However, there are 14 unknown variables (six torques, four components for the internal forces in both knees and four components for both ground reactions), for 13 scalar equations only. Consequently, we have to prescribe one unknown variable as parameter. To 
highlight this point let us consider the global equilibrium in translation and rotation of the biped (18), with Fig.5. The center of mass $G$ of the biped is located with the coordinates $x_{g}$ and $y_{g}, \mathbf{r}_{1}$ and $\mathbf{r}_{2}$ are the ground reaction acting in the front hell and the rear toe, $\delta_{g}$ is the dynamic momentum of the biped with respect to its center of mass. We have four unknown variables, $r_{1 x}, r_{2 x}, r_{1 y}$ and $r_{2 y}$ for three equations only.

$$
\left\{\begin{array}{c}
y_{g}\left(r_{1 x}+r_{2 x}\right)+\left(d-x_{g}\right) r_{2 y}+x_{g} r_{1 y}=\delta_{g} \\
r_{1 x}+r_{2 x}=m \ddot{x}_{g} \\
r_{1 y}+r_{2 y}-m g=m \ddot{y}_{g}
\end{array}\right.
$$

If the sum $r_{1 x}+r_{2 x}$ is unique, there are an infinity of solutions for $r_{1 x}$ or $r_{2 x}$ which satisfy (18). As a

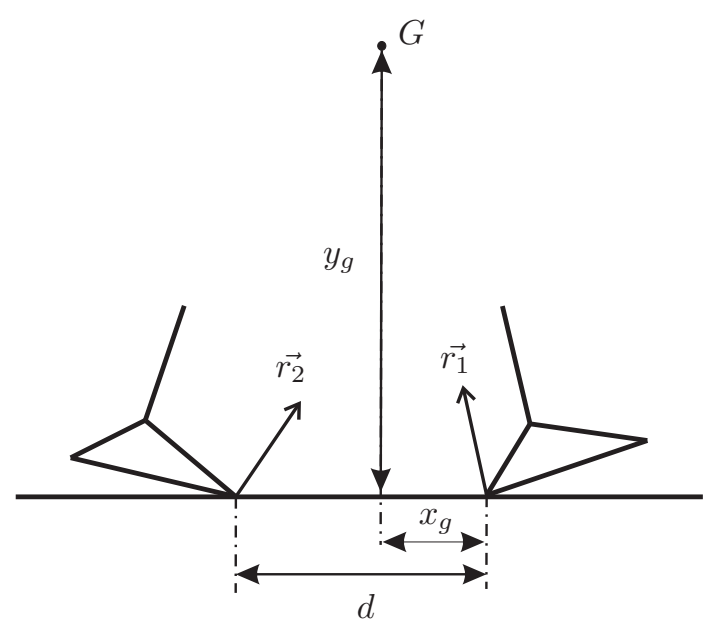

Figure 5: Ground reactions in double support phase and the center of mass of the biped.

consequence $r_{2 x}$ can be defined as a second order polynomial function in time:

$$
r_{2 x}(t)=d_{0}+d_{1} t+d_{2} t^{2}
$$

The number of parameter is equal to 29.

$$
\begin{array}{r}
P=\left[q_{1}\left(T_{D S}\right), q_{2}\left(T_{D S}\right), q_{5}\left(T_{D S}\right), q_{p 2}\left(T_{D S}\right), q_{p 2}\left(\frac{T_{S S}}{2}\right), q_{1}\left(\frac{T_{S S}}{2}\right), q_{2}\left(\frac{T_{S S}}{2}\right), q_{3}\left(\frac{T_{S S}}{2}\right), q_{4}\left(\frac{T_{S S}}{2}\right),\right. \\
q_{5}\left(\frac{T_{S S}}{2}\right), \dot{q}_{p 1}\left(T_{D S}\right), \dot{q}_{p 2}\left(T_{D S}\right), \dot{q}_{1}\left(T_{D S}\right), \dot{q}_{2}\left(T_{D S}\right), \dot{q}_{5}\left(T_{D S}\right), x_{h}\left(T_{S S}\right), y_{h}\left(T_{S S}\right), \\
\left.q_{5}\left(T_{S S}\right), d, \dot{q}_{p 2}\left(T_{S S}\right), \dot{q}_{1}\left(T_{S S}\right), \dot{q}_{2}\left(T_{S S}\right), \dot{q}_{3}\left(T_{S S}\right), \dot{q}_{4}\left(T_{S S}\right), \dot{q}_{5}\left(T_{S S}\right), d_{0}, d_{1}, d_{2}, T_{D S}\right]
\end{array}
$$

The variables $T_{S S}$ and $T_{D S}$ are the duration of the single support and the double support respectively for the cyclic walking gait 2 .

\subsection{Parametric optimization problem}

Basis functions as polynomial functions, see [4], truncated Fourier series, see [3] or cubic spline function, see [31] can be chosen for the parametric optimization algorithm. A basis function of high order can lead to oscillatory phenomenon during the optimization process, see see [6] or [21]. By parameterizing the joint motion in terms of cubic spline functions, the optimization problem is reduced to a constrained parameter optimization problem of the form:

$$
\begin{array}{cl}
\text { Minimize } C_{W} & (\mathbf{P}) \\
\text { subject to } \mathbf{g}_{\mathbf{j}} & (\mathbf{P}) \leq 0 \text { for } j=1,2, \ldots l
\end{array}
$$

where $\mathbf{P}$ is the set of optimization variables. $C_{W}(\mathbf{P})$ is the criterion to minimize with $l$ inequality constraints $\mathbf{g}_{j}(\mathbf{P}) \leq 0$ to satisfy. Cubic function of third-order are chosen for the single and double support phase with two selected knots. Thus the cubic spline are uniquely defined by specifying an initial configuration, an initial velocity, a final configuration and a final velocity. The criterion and constraints are given in the following sections.

We used the SQP method (Sequential Quadratic Programming) [17], [29] with the fmincon function

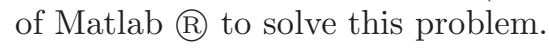




\subsubsection{The criterion}

Many criteria can be used to produce an optimal trajectory. A sthenic criterion is chosen to obtain optimal trajectories:

$$
C_{W}=\frac{1}{d} \int_{0}^{T} \sum_{i=1}^{6} \Gamma_{m_{i}}^{\mathrm{t}} \Gamma_{m_{i}} \mathrm{~d} t
$$

where $T$ is the step duration and $\Gamma_{m_{i}}$ is the torque applied in joint $i$. During an optimization process the step length $d$ is an optimization variable and the walking speed $v$ is fixed, such as the step duration is directly given through the relation $T=d / v$.

The resulting optimal control is continuous and cancels the risks of a jerky functioning [7]. This smoothness property also guarantees a better numerical efficiency for the algorithm used for the optimization problem-solving

\subsubsection{The constraints}

Two types of constraints are used to obtain a realistic gait.

- The necessary constraints, which ensure a valid walking gait.

The first constraint ensures the supporting leg tip does not take off or slide on the ground. So, the ground reaction force is inside a friction cone, defined with the coefficient of friction $f$ :

$$
\left\{\begin{array}{l}
\max \left(-f r_{i y}-r_{i x}\right) \leq 0 \\
\max \left(-f r_{i y}+r_{i x}\right) \leq 0
\end{array}\right.
$$

$j=1$ or $2 . r_{x}$ and $r_{y}$ are the normal and tangential component of the reaction force. Moreover, we can introduce a constraint on the ground reaction at the impact:

$$
\left\{\begin{array}{l}
\left(-f i_{1 y}-i_{1 x}\right) \leq 0 \\
\left(-f i_{2 y}+i_{2 x}\right) \leq 0
\end{array}\right.
$$

To ensure the no rotation of the supporting foot we introduce a constraint on the $Z M P$ during the single support phase and at the instant of the impact:

$$
\left(l_{p}-L_{p}\right) \leq l_{Z M P} \leq l_{p}
$$

Here $L_{p}$ is the length of the foot and $l_{p}$ is the distance between the heel and the ankle along the horizontal axis, see Fig. 3.

Just after the impact, the velocity of the taking-off foot should be directed upward. In consequence, the positivity of the vertical component of the velocities for the heel and the toes is added to the set of constraints.

The last constraint allows to ensure the non penetration of the swinging foot in the ground.

- The unnecessary constraints, which ensure a technological realistic gait.

We introduced mechanical stops on the joint variables. Moreover, we limited the torques with a constraint, which sets a template of the maximum torque of the motor relatively to the velocity [5].

\section{Results}

With this optimization process, a set of optimal trajectories is developed for different walking velocities for a biped equipped of four-bar knees. We use for the $S Q P$ algorithm the fmincon function of Matlab $®$ The $S Q P$ algorithm solves a local optimization problem. However we tried success different initial conditions, which satisfy the constraints defined in the previous section 3.2.2. We can see Fig. 6.a a comparison of the sthenic criterion according to the walking velocities for the biped with the four-bar knees and with the revolute joint knees. Despite the local optimization problem we can see the the four-bar knees offer a benefice with respect to the sthenic criterion: for all the walking velocities the sthenic criterion is bigger with the revolute joint knees than with the four-bar knees. For the biped with the four-bar knees we can see Fig. 7.a the ratio between the part of the sthenic criterion during the double support phase and the sthenic criterion and between the part of the sthenic criterion during the single support phase and the sthenic criterion. For the slow walking gaits the most part of the sthenic criterion is consumed during 
the double support phase. For the fast walking gaits the ratio of the part of the sthenic criterion between the single support phase and the sthenic criterion increases. It is logical from a physical point of view. The parts of the sthenic criterion consumed by the biped during the double support and single support phases are quasi equal for around a velocity of $4 \mathrm{Km} / \mathrm{h}$. For walking velocities between $1.5 \mathrm{Km} / \mathrm{h}$ and $2.5 \mathrm{Km} / \mathrm{h}$ the motion of the transfer leg with a four-bar knee is quasi passive, Fig. 7.b. Its consumed part of the sthenic criterion is very small with respect to the sthenic criterion. It is one of the remarkable characteristics of the walking gaits for human [13]. We do not observe this property with a walking gait, which is only composed of single support phases and impacts, see [20]. Then it is interesting to insert a double support phase to come closer to the anthropomorphic walking gait.

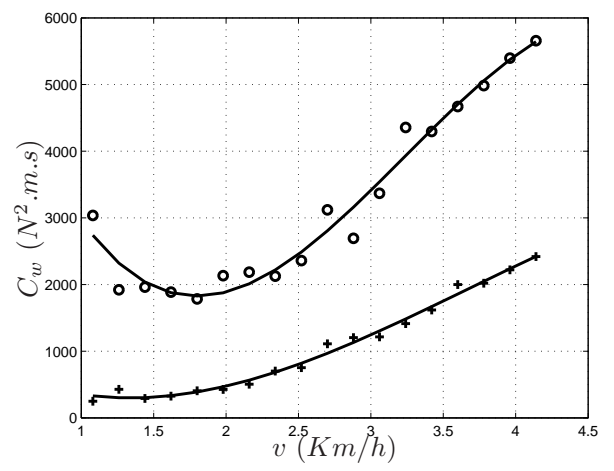

(a)

Figure 6: a): Evolution of the sthenic criterion of bipedal robot according to the walking velocity, (o) revolute joint knees, $(+)$ four-bar knees.

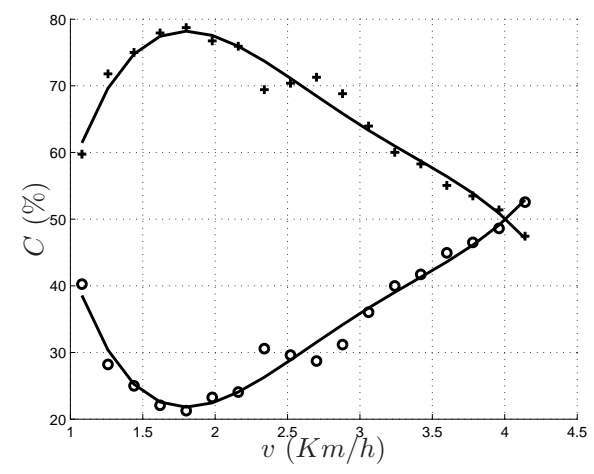

(a)

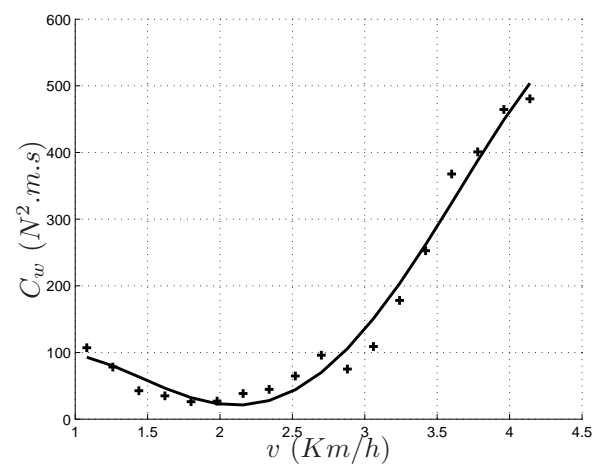

(b)

Figure 7: a): Evolution of the sthenic criterion of bipedal robot according to the walking velocity, (o) revolute joint knees, $(+)$ four-bar knees. a) Ratio between the part of the sthenic criterion during the double support phase and the sthenic criterion (plus marker) and between the part of the sthenic criterion during the single support phase and the sthenic criterion (round marker) as a function of the walking velocity. b) Evolution of the part of the sthenic criterion for the transfer leg.

Figure 8.a presents the evolution of the double support and single support duration during a walking gait as a function of the walking velocity. Figure 8.b gives the evolution of the step length as a function of the walking velocity. From Fig. 8.a and Fig. 8.b we observed that, when the walking velocity increases, the duration of the single support phase decreases. The durations of the double support phase and the single support phase tend to be equal when the walking velocity increases. 


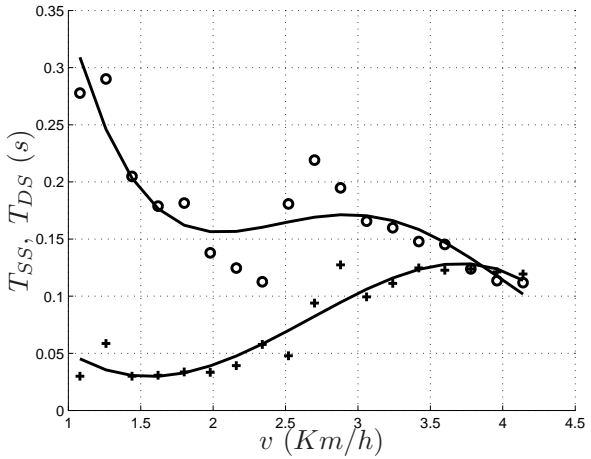

(a)

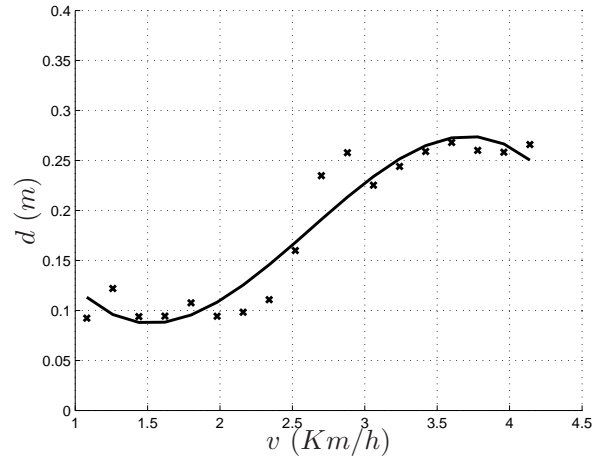

(b)

Figure 8: a): Evolution of the double support phase duration (plus marker) and the single support phase duration (round marker) as a function of the walking velocity. b) Evolution of the step length as a function of the walking velocity.

Figure 9.a ${ }^{1}$ shows the flexion and the extension movements according to the time measurement of the left knee in the sagittal plane during the same cycle of a walking male of a height of $1.7 \mathrm{~m}$. Figures 9.b and 9.c show the vertical sides of the heel and big toe of the left foot. The balancing phase takes place between $0.6 \mathrm{~s}$ and $0.9 \mathrm{~s}$. For this cycle both feet are grounded for $0-15 \%$ of the cycle.

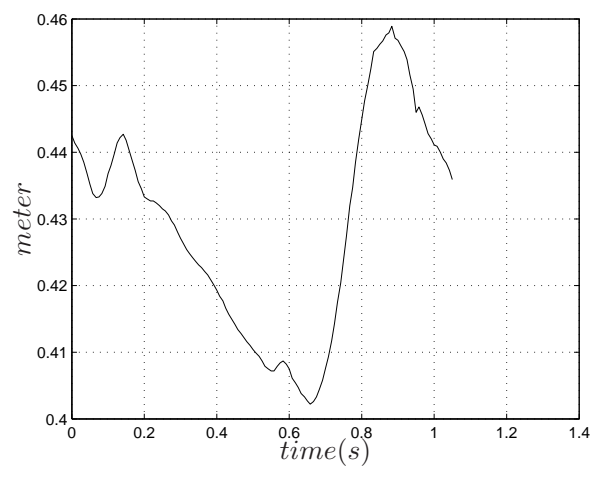

(a)

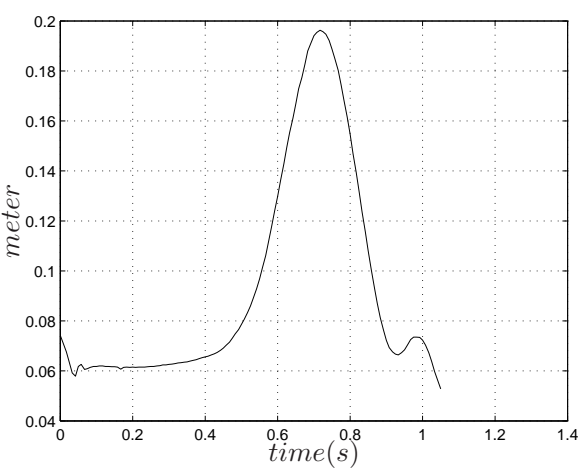

(b)

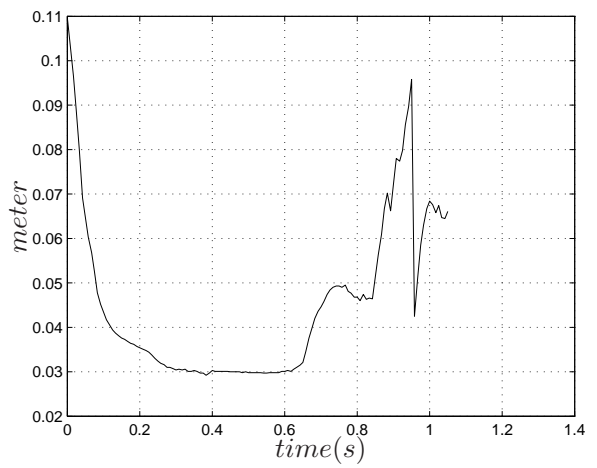

(c)

Figure 9: a): Flexion and extension of the left knee according to the time measurement of the left knee. b) Vertical side of the heel according to the time measurement. c) Vertical side of the big toe according to the time measurement.

\footnotetext{
${ }^{1}$ The following measurements were communicated to us by Franck Multon, who is part of the Physiology and Muscular Exercise Laboratory of the University of Rennes 2 and the ENS of Cachan
} 
Figures 10 are devoted to the leg, which is the swing leg during the single support. Figure 10.a shows the flexion and the extension movements according to the time measurement of the four-bar knee in the sagittal plane during the step of a walking gait. Figures 9.b and 9.c show the vertical sides of the heel and big toe of the foot. The balancing phase takes place between $0.4 \mathrm{~s}$ and $0.6 \mathrm{~s}$. Of course there are many differences between the results from the numerical test and the human data, specially for the knee joint. However despite that the size of the walking man is different from those of the bipedal robot the simulated walking gait leads to similar scales of size with respect to human.

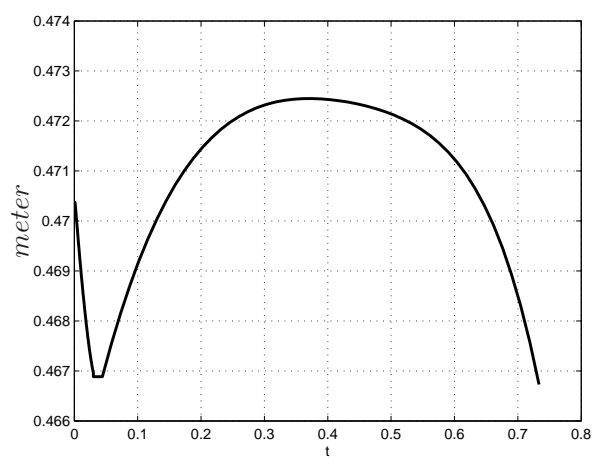

(a)

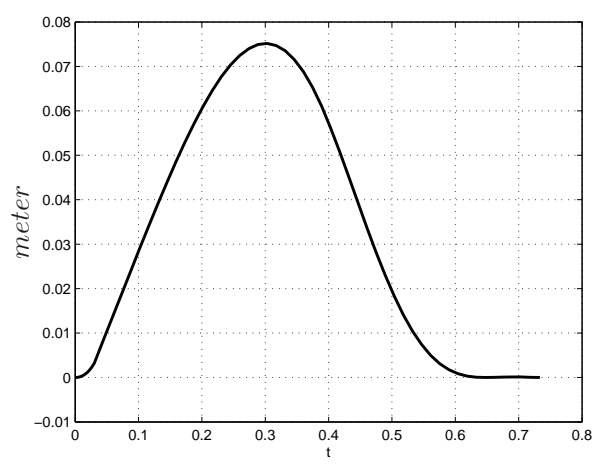

(b)

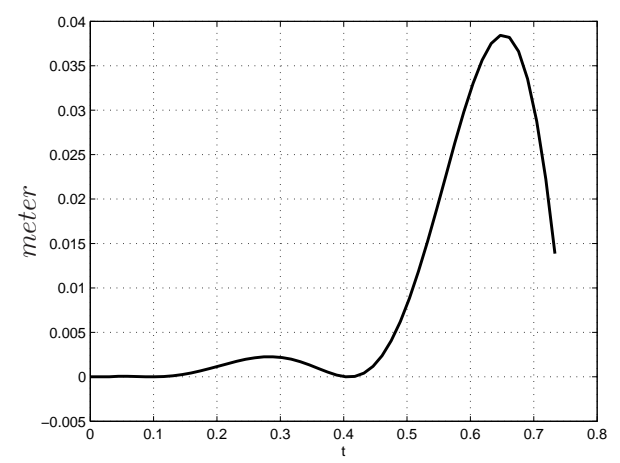

(c)

Figure 10: a): Flexion and the extension of the four-bar knee according to the time measurement. b) Vertical side of the heel according to the time measurement. c) Vertical side of the big toe according to the time measurement.

\section{Conclusions}

We have studied a closed-loop linkage for the knees of a planar bipedal robot. We have produced with a parametric optimization method a set of optimal reference walking trajectories for this bipedal robot. The walking motions are composed of single support and double support phases separated by impulsive impact. The benefice on the sthenic criterion of a four-bar knee is real with respect to the revolution joint because the needed torques for a similar walking gaits are smaller with the four-bar knees. Then in practice, it is possible to reduce the size of the actuators and to decreased the energy consumption. The study of the sthenic criterion of this biped has shown interesting results in term of energy distribution, between the single support phase and the double support phase, as a function of the walking velocity, which can be compared with the characteristics of the human walking gait. The characteristics of the walking gait of the bipedal robot equipped with four-bar knees are still far from those of human. But the human walking gait is more complex with a rolling sole on the ground of the stance foot than our defined walking gait. Furthermore a great attention about the optimal dimensions of the four-bar mechanism is necessary.

The perspective of this study is to extend this work for a 3D bipedal robots and to test this new structure with a experimental bipedal robots. Moreover, a measure of the evolution of the center of 
rotation of the human knee will be utilized to choose with a better accuracy the dimensions of the fourbar mechanism. Finally, a single support phase with rotation of the support foot can be introduced to come closer to the results of an anthropomorphic walking motion.

\section{References}

[1] Paul Appell. Dynamique des Systèmes - Mécanique Analytique. Gauthiers-Villars, 1931.

[2] J. Bradley, D. FitzPatrick, D. Daniel, T. Shercliff, and J. O'Connor. Orientation of the cruciate ligament in the sagittal plane. The Journal of bone and joint surgery, 70-B:94-99, 1988.

[3] G. Cabodevilla, N. Chaillet, and G. Abba. Near optimal gait for a biped robot. In Proceedings of the $A M S^{\prime} 95$ 1995, Kalrsuhe, Germany, 1995.

[4] C. Channon, S. H. Hopkins, and D. T. Pham. Derivation of optimal walking motions for a bipedal walking robot. Robotica, 2(14):165-172, 1992.

[5] C. Chevallereau, G. Abba, Y. Aoustin, F. Plestan, E.R. Westervelt, C. Canuddas-de Wit, and J.W. Grizzle. Rabbit: a testbed for advanced control theory. IEEE Control Systems Magazine, 23(5):5779, 2003.

[6] C. Chevallereau and Y. Aoustin. Optimal reference trajectories for walking and running of a biped robot. Robotica, 19(5):557-569, 2001.

[7] C. Chevallereau, G. Bessonnet, G. Abba, and Y. Aoustin. Bipedal Robots. ISTE Wiley, 2009.

[8] S. L. Delp, J. P. Loan, M. G. Hoy, F. E. Zajac, E. L. Topp, and J. M. Rosen. An interactive graphicsbased model of the lower extremity to study orthopaedic surgical procedures. IEEE Transactions on Biomedical Engineering, 37(8):757-767, 1990.

[9] S.F Dye. An evolutionary perspective of the knee. The Journal of Bone and Joint Surgery, 69(7):976983, 1987.

[10] N. Farhat, V. Mata, D. Rosa, J. Fayos, and X. Peirau. "musculo-skeketic model for knee joint forces estimation in sport activities". In 7th EUROMECH Solid Mechanics Conference, Lisbon, Portugal, 7-11 September 2009.

[11] N. Farhata, V. Mata, D. Rosab, and J. Fayos. A procedure for estimating the relevant forces in the human knee using a four-bar mechanism. Computers Methods in Biomechanics and Biomedical Engineering, 13(5):577-587, 2010.

[12] J. D. Feikes, J. J. O'Connor, and A. B. Zavatsky. A constraint-based approach to modelling the mobility of the human knee joint. Journal of Biomechanics, 36(1):125 - 129, 2003.

[13] A.M. Formalskii. Locomotion of Anthropomorphic Mechanisms. [In Russian], Nauka, Moscow, Russia, 1982.

[14] F. Freudenstein and L.S. Woo. Kinematics of human knee joint. Bulletin of Mathematical Biophysics, $31(2): 215-232,1969$.

[15] Frank K. Fuss. Anatomy of the cruciate ligaments and their function in extension and flexion of the human knee joint. American Journal of Anatomy, 184(2):165-176, 1989.

[16] Steven A. Gard, Dudley S. Childress, and Jack E. Uellendahl. The influence of four-bar linkage knees on prosthetic swing-phase floor clearance. Journal of Prosthetics and Orthotics, 8(2):34-40, 1996.

[17] P.E. Gill, W. Murray, and M.H. Wright. Practical optimization. Academic Press, London, 1981.

[18] G. Gini, U. Scarfogliero, and M. Folgheraiter. Human-oriented biped robot design : insights into the development of a truly antropomophic leg. In IEEE International Conference on Robotics and Automation, pages 2910-2915, 2007.

[19] A.A. Grishin, A.M. Formal'sky, A.V. Lensky, and S.V. Zhitomirsky. Dynamic walking of a vehicle with two telescopic legs controlled by two drives. The International Journal of Robotics Research, 13(2):137-147, 1994. 
[20] Arnaud Hamon and Yannick Aoustin. Cross four-bar linkage for the knees of a planar bipedal robot. In 2010 IEEE-RAS International Conference on Humanoid Robots, pages 379-384, 2010.

[21] L. Hu, C. Zhou, and Z. Sun. Biped gait optimization using spline function based probability model. in Proceedings of the IEEE Conference on Robotics and Automation, pages 830-835, 2006.

[22] S. Kajita, K. Kaneko, M. Morisawa, S. Nakaoka, and H. Hirukawa. Zmp-based biped running enhanced by toe springs. In 2007 IEEE International Conference on Robotics and Automation, pages 3963-3969, 2007.

[23] S. Kajita, T. Nagasaki, K. Kaneko, K. Yokoi, and K. Tanie. A running controller of humanoid biped hrp-2lr. In Proceedings of the IEEE International Conference on Robotics and Automation 2005, volume 18-22, pages 616-622, 2005.

[24] W. Khalil and E. Dombre. Modeling, identification and control of robots. Hermes Sciences Europe, 2002.

[25] H. Kurosawa, P. S. Walker, S. Abe, A. Garg, and T. Hunter. Geometry and motion of the knee for implant and orthotic design. Journal of Biomechanics, 18(7):487-491, 1985.

[26] B. Landjerit and M. Bisserie. Cinématique spatiale de l'articulation fémoro-tibiale du genou humain: caractérisation expérimentale et implications chirurgicales. Acta Orthopaedica Belgica, 58(2):147$158,1992$.

[27] A. Leardini, J.J. O'Connor, F. Catani, and S. Giannini. A geometric model of the human ankle joint. Journal of Biomechanics, 32(6):585 - 591, 1999.

[28] A. Menschik. Mechanics of the knee-joint. part i. Z Orthop Ihre Grendgeb, 112(3):481-495, 1974.

[29] M.J.D. Powell. Variable metric methods for constrained optimization, pages 62-72. Lecture Notes in Mathematics. Springer Berlin / Heidelberg, 1977.

[30] R. Tajima, D. Honda, and K. Suga. Fast running experiments involving a humanoid robot. In 2009 IEEE Conference on Robotics and Automation, pages 1571-1576, 2009.

[31] D. Tlalolini Romero, Y. Aoustin, and C. Chevallereau. Design of a walking cyclic gait with single support phases and impacts for the locomotor system of a thirteen-link $3 \mathrm{~d}$ biped using the parametric optimization. Multibody System Dynamics, 23(1):33-56, 2009.

[32] D. Tlalolini Romero, C. Chevallereau, and Y. Aoustin. Comparison of different gaits with rotation of the feet for a planar biped. Robotics and Autonomous Systems, 57(4):371-383, Avril 2009.

[33] M. Vukobratovic and J. Stepanenko. On the stability of anthropomorphic systems. Mathematical Biosciences, 15(1):1-37, 1972.

[34] F. Wang, C. Wu, Y. Zhang, and X. Xu. Design and implementation of coordinated control strategy for biped robot with heterogeneous legs. In IEEE International Conference on Mechatronics and Automation, pages 1559-1564, 2007.

[35] D. R. Wilson, J. D. Feikes, and J.J. O'Connor. Ligaments and articular contact guide passive knee flexion. Journal of Biomechanics, 31:1127-1136, 1998.

[36] T. Yang, E. Westervelt, J. Schmideler, and R. Bockbrader. Design and control of a planar bipedal robot ernie with parallel knee compliance. Autonomous Robots, 25:317-333, 2008. 